\title{
There is no such thing as "twins" Mini-commentary on BJOG-20-0566.R2
}

\author{
C Andrew Combs ${ }^{1}$ \\ ${ }^{1}$ Obstetrix Medical Group
}

September 10, 2020

Tweetable abstract: Identical twin pregnancies have more preterm births and other complications than fraternal twin pregnancies.

\section{Mini-Commentary}

A decade ago, Professor Kypros Nicolaides of Kings' College opined, "There is NO diagnosis of twins. There are only monochorionic or dichorionic twins. This diagnosis should be written in capital red letters across the top of the patient's chart." (Quoted by Moise and Johnson, Am J Obstet Gynecol 2010; 203:1-2.) To make this diagnosis, it is essential to establish chorionicity as early as possible in every twin pregnancy.

Monochorionic twin pregnancies have long been known to have higher rates of miscarriage, congenital anomalies, stillbirth, and neonatal death than dichorionic twin pregnancies. Intertwin vascular anastomoses are present in most monochorionic twin placentas, leading to complications such as twin-twin transfusion syndrome (TTTS), twin anemia-polycythemia sequence (TAPS), twin reversed arterial perfusion (TRAP) sequence, and unequal placental sharing (UPS).

Monochorionic twins require intensive antenatal surveillance. Because of the increased risk of congenital anomalies, fetal echocardiogram is recommended in addition to routine ultrasound fetal anatomy survey. Because of the risk of TTTS, TAPS, TRAP and UPS, sonographic surveillance is recommended every 2 weeks starting at 16 weeks of gestation. Because of the risk of stillbirth, serial antenatal cardiotocography is recommended. Scheduled delivery is recommended earlier for monochorionic twins than for dichorionic twins (NICE Guideline 137, 2019; ACOG, Obstet Gynecol 2019;133:e151-5; Cheong-See et al, BMJ 2016;354:i4353).

Regardless of chorionicity, $60 \%$ of twins are born preterm, resulting in substantial perinatal morbidity and mortality. Prevention of preterm birth (PTB) is a major priority for management of twin pregnancy.

The systematic review by Marleen and colleagues is the first of several studies planned by the authors to evaluate risk factors for PTB in twin pregnancy. Prior reports have suggested that monochorionic twins have higher rates of PTB than dichorionic twins but, as the authors note, there has been no prior systematic review of this association. It is not surprising that the review shows an increased overall rate of PTB among monochorionic twins in all gestational age ranges, given that complications such as stillbirth, TTTS, TAPS, TRAP, and UPS often result in iatrogenic PTB. Indeed, iatrogenic PTB before 37 weeks of gestation should be routine for monochorionic twins because of the increasing risk of stillbirth past $36^{+6}$ weeks cited in the current NICE Guidelines (2019, op. cit .). However, Marleen and colleagues also report that spontaneous PTB at $<37$ weeks and [?] 34 weeks is increased in monochorionic twin pregnancy, which cannot be directly explained by monochorionic placental complications.

The overarching goal of Marleen and colleagues is to develop tools to predict which twin pregnancies are at risk of PTB so that preventive measures can be taken. Unfortunately, it is not currently known what preven- 
tive measures will reduce the high risk of early spontaneous PTB among monochorionic twin pregnancies. Prophylactic bedrest, hospitalization, uterine activity monitoring, tocolysis, cerclage, cervical pessary, and progestogens have not proven effective for unselected twin pregnancies. Future research will be needed to determine the value of such interventions for women with twin pregnancy plus additional risk factors such as a short cervix, prior PTB, or monochorionicity.

Acknowledgements: None

Disclosure of Interests: C Andrew Combs declares "No relevant or competing interests"

Contribution to Authorship: CAC did 100\% of the planning, writing, and submission.

Details of Ethics Approval : Not applicable

Funding: None

References: Cited in-line per instructions for Mini-Commentary

Tables/Figures: None 